\title{
Critérios para a Determinação dos Investimentos mais Econômicos Computadores Digitais
}

Este estudo será dividido nas seguintes partes:

1. Colocação do problema

2. Solução do problema

3. Demonstração do critério empregado na solução, onde será mostrada a sua validade

4. Utilização do computador digital: a) apresentação do programa, isto é, do plano para a sua realização; b) entrada e saída de dados; c) o programa pròpriamente dito, contendo, além da listagem, o exemplo de um processamento de dados efetuado

5. Considerações finais.

1. COLOCAÇAO DO PROBLEMA

Sejam dados vários planos de investimentos por meio dcs correspondentes fluxos líquidos de caixa (net cash-flow) e a taxa mínima atrativa de retôrno.

Deseja-se ordenar êsses planos segundo o critério do máximo lucro e saber qual o lucro obtido com cada um dêles.

\section{SOLUÇAOO DO PROBLEMA}

Consideremos um plano de investimento, dado pelo seguinte fluxo de caixa líquido:

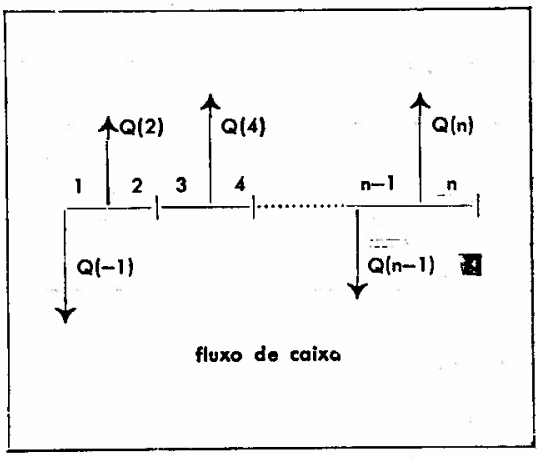

Os valôres $Q(1), Q(2), \ldots$, $Q(n-1), Q(n)$, representam cada montante em dinheiro, e todos êles, das entradas e saidas de caixa decorrente da aplicação

* Professor do Departamento de Métodos Quantitativos da Escola de Administraçâo de Emprêsas de São Paulo, da Fundação Getúlio Vargas. 
dêsse plano, serão positivos (para cima) se fôr entrada de dinheiro (reembôlso) e negativos (para baixo), como $Q(1)$, $Q(n-1)$ se significar saída (desembôlso ou investimento.) Os números $1,2, \ldots, n-1, n$, associados aos intervalos, são os períodos de tempo durante a execuçãa do plano, dados em meses, anos, etc.; todos os períodos são iguais e $Q(i)$, $i=1,2, \ldots, n$, pode ser nulo ou não, exceto $Q(1)$ que deverá ser negativo, isto é, supõe-se que todo plano se inicia com desembôlso de dinheiro. 0 número $\mathbf{n}$ indica o número de períodos em que. o plano é aplicado e deve abranger o plano desde o seu início até o seu fim.

Ilustraremos, o acima exposto, com um exemplo, onde os dados podem ser os previstos, no caso de um planejamento, ou os passados, no caso de se pretender fazer uma análise do que já foi realizado.

Seja, então, a compra de uma propriedade por $\operatorname{Cr} \$ 200 \mathrm{mil}$, no início de 1970, que irá render Cr\$100 mil (receita menos despesa, ou lucro) ao ano até 1972, sendo vendida no início de 1973 por Cr\$100 mil. (Este exemplo foi processado num computador IBM-1130, e é o plano 3; ver a seção 4.3).

Neste caso, o fluxo de caixa será:

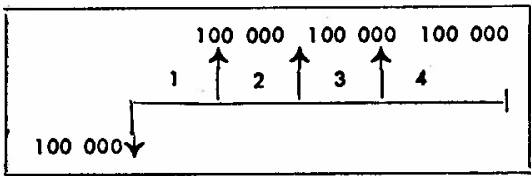

isto é,

$Q(1)=\$-100000,00$

$Q(3)=\$+100000,00$

$Q(2)=\$+100000,00$ $Q(4)=\$+100000,00$

e $n=4$ (períodos de um ano cada). Note-se a convenção do pagamento no início do período (antecipado).

Seja $R_{m i n}$ a mínima taxa de retôrno admitida pela política econômica da emprêsa que es- tuda os investimentos (por exemplo, $R_{\min }=15 \%$ a/a ou $\left.0,15 \mathrm{o} / 1^{*} \mathrm{a} / \mathrm{a}\right)$.

A partir dêsses dados, $Q(1), \ldots$, $Q(n)$ podemos determinar a taxa de retôrno do investimento, que será tal que a projeção dos desembolsos no presente, ou valor presente, seja igual ao valor presente dos reembolsos. No exemplo acima, seria um $\mathbf{R}$ tal que:

$$
\begin{gathered}
-Q(1)=100.000=100.000 \\
{\left[(1+R)^{-1}+(1+R)^{-2}+(1+R)^{-3}\right]}
\end{gathered}
$$

Êsse cálculo fornece (conforme seção 4.3)

$$
R=0,83928 \text { o/ } 1 \text { ou } 83,928 \% \text {. }
$$

Conhecido $\mathbf{R}$, determina-se o lucro total por período (a menos de uma constante, cf. seção 3) como sendo (A é o valor presente dos investimentos):

$$
V=\left(R-R_{\min }\right) A \text {. }
$$

No exemplo anterior,

$A=100.000,00 R=0,83928 / 1$

$\mathrm{R}_{\min }=0,15 . / 1 \mathrm{e} \mathrm{V}=100.000$ $(0,83928-0,15)=\$ 68.928$.

Essa mesma seqüência deverá ser repetida para os vários planos apresentados. No final, teremos vários valôres de. V:

$V(1), V(2), \ldots \ldots \ldots, V(S)$, onde $s$ é o número de planos.

O melhor plano (ou os melhores) será aquêle que fornecer o maior valor a $\mathrm{V}(\mathrm{j})$, onde j é um dos planos.

\section{DEMONSTRAÇÃO DO CRITÉRIO}

Quanto ao conceito de taxa de retôrno, o utilizado neste trabalho é o que resulta da equivalência de séries de pagamentos, que supomos conhecido. ${ }^{1}$

Deter-nos-emos no critério da escolha do melhor plano, segundo um estudo por nós realizado.

Admitamos que se disponha de um capital C para ser aplicado nos melhores planos entre os apresentados. Seja $A(j)$ o valor presente dos investimentos, onde $\mathbf{j}$ é o plano considerado $(j=1,2, \ldots, s)$. Se o único desembôlso fôr o inicial, $A(J)$ coincide com o valor dêsse reembôlso.

Seja, então, aplicado o montante $A(j)$ no plano $j$, que será a juros de $R(j)$ (igual a $83,9 \%$ no exemplo anterior), e o restante que se dispõe, $C-A(j)$, a juros de $R_{\min }(15 \%$, no exemplo anterior).

Se o valor de revenda fôr igual ao do investimento, isto $k$, se não houver depreciação durante a utilização do plano (e esta hipótese não altera a generalidade da análise ${ }^{2}$ o lucro equivalente por período será:

$$
\begin{gathered}
A(j) R(j)+(C-A(j)) R_{\min } \text { ou } \\
C R_{\min }+A(j)\left(R(j)-R_{\min }\right)
\end{gathered}
$$

Como nossa finalidade é maximizar o lucro, devemos achar o plano (isto é, o valor de j) que nos dará o maior valor à expressão acima. Mas nessa expressão, $\mathrm{CR}_{\min }$ não depende de j; basta, então, maximizar:

$V(j)=\left(R(j)-R_{m(n)}\right) \cdot A(j)$

que é o algoritmo por nós usado (q.e.d.).

\section{UTILIZAÇÃO DO COMPUTADŌR DIGITAL}

\subsection{Apresentação do programa.}

O programa se encontra dividido em duas partes: a primeira calcula a taxa de retôrno, cujo algoritmo utilizado, próprio para computadores, foi desenvolvido por Savir e Sherman; ${ }^{3}$ a segunda seleciona os melhores planos, usando 0 algoritmo já exposto.

\footnotetext{
* o/1 significa por unidade.

1 Grant. Principles of engeneering economy. 4. ed., New York, The Ronald Press, 1964. cap. 8.

Id. ibid. cap. 12.

3 DeGarmo. Engeneering economy. 4. ed., New York, The MacMillan Co., 1969. apêndice $c$.
} 
Damos, a seguir, os diagramas

de bloco correspondente a essas

duas partes, que esclarecem

o fluxo de informações en-

volvido.
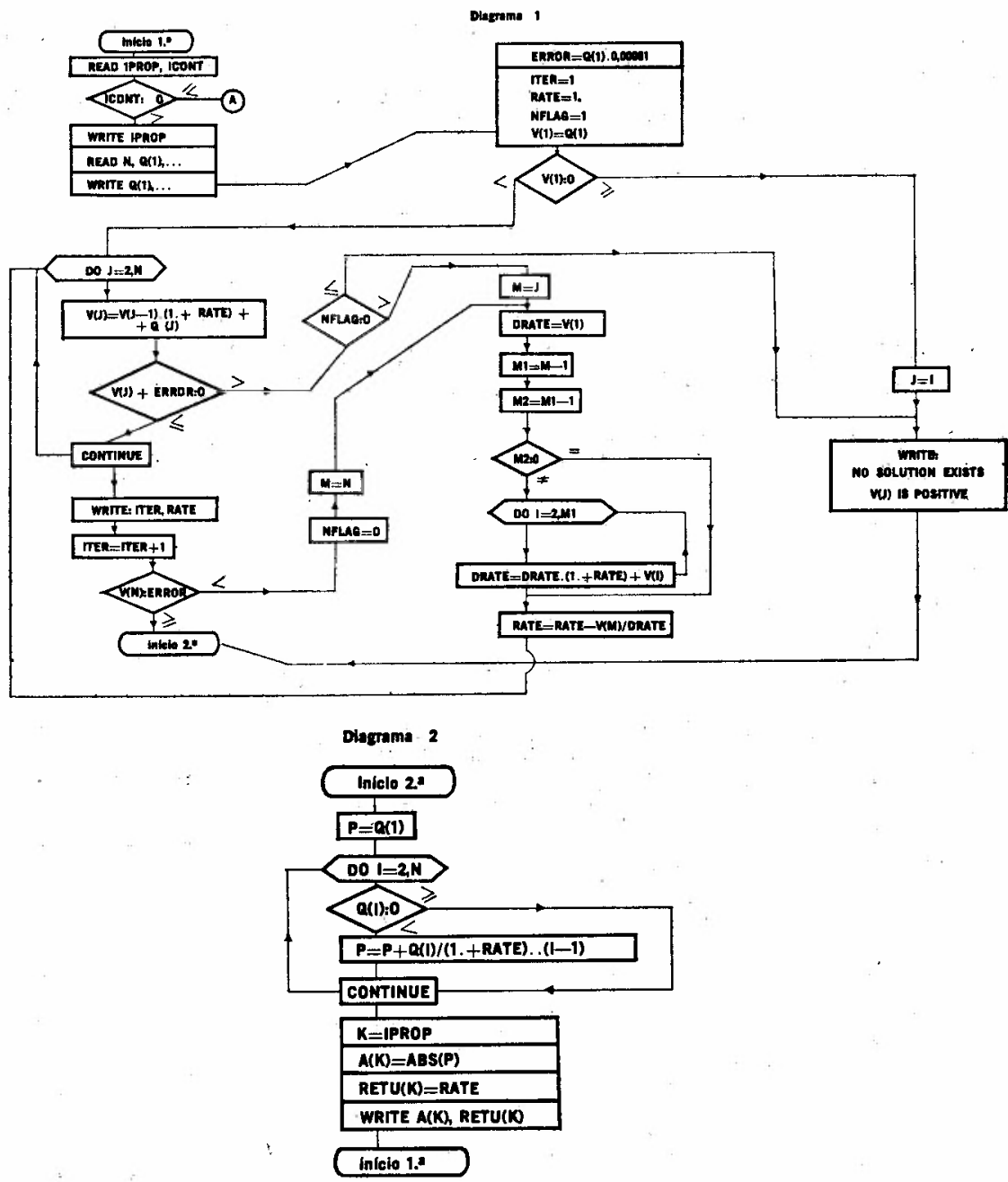

(A)

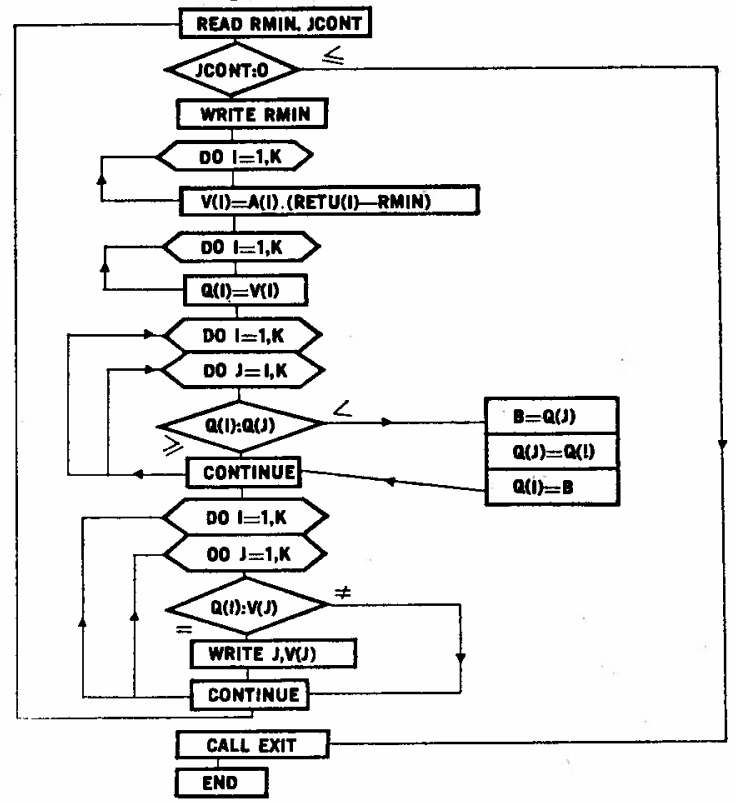

\subsection{Entrada e saída de dados}

Todos os cartões possuem um campo de 10 colunas, onde deverão ser perfurados os números.

Após o cartão //XEQ, o primeiro cartão deverá ter o n. ${ }^{\circ} 1$ perfurado na $10 .^{a}$ coluna, indicando que a seguir virão os dados do primeiro plano; e o n. 1 na 20. a coluna, indicando que haverá um plano a ser lido a seguir. Estes serão os valôres de IPROP e ICONT, respectivamente.

O segundo cartão terá impresso no campo, de 0 até a $10 .^{a}$ coluna, o número de períodos (N), do plano em questão. Se fôr, por exemplo, 11 períodos, terá perfurado um na nona coluna e um na $10 .^{a}$.

O terceiro cartão terá os valôres de $Q(1)$, sendo seis valôres por cartão, com campos de 10 colunas cada (vírgula decimal entre a $10 .^{a}$ e $11 .^{a}$ coluna), que prosseguirá no quarto cartão, etc., até completar $\mathbf{N}$ valóres de $Q(I)$. Note-se que $Q(1)$ deverá ser sempre negativo.

Depois, repete-se o ciclo para o próximo plano, sendo que, como no primeiro cartão, deverá estar, também, perfurado um na $20 .^{a}$ e no n. 2 na $10 .{ }^{a}$. E assim por diante, até completarem-se todos os planos. Deverá haver, então, um cartão em branco, indicando que o próximo cartão fornecerá o valor da taxa mínima atrativa (RMIN) por unidade (o/1), com o ponto decimal entre a quinta e a sexta colunas e situado no campo das 10 primeiras colunas. $\mathrm{Na} 20 .^{\mathrm{a}}$ dêsse cartão deverá haver o n.० 1 , indicando que êsse dado deverá ser processado.

Outros cartões com outras RMIN poder-se-ão seguir (todos com o um perfurado na 20.a) $\mathrm{E} O$ último cartão de dados deverá ser, necessàriamente, virgem (em branco, não perfurado). 
Quanto à saída de dados, se não existir uma taxa de retôrno num plano, isso será impresso pela impressora 1132. As colunas period e cash flow indicam os valôres lidos do fluxo de caixa (dados de entrada). As iteration e rate of return mostram a convergência do algoritmo usado. Na coluna algorithm estão os valôres de $V(I)$.

4.3 O Programa pròpriamente dito

Esse programa está redigido em linguagem FORTRAN IV - Monitor, para o computador I.B.M. 1130.

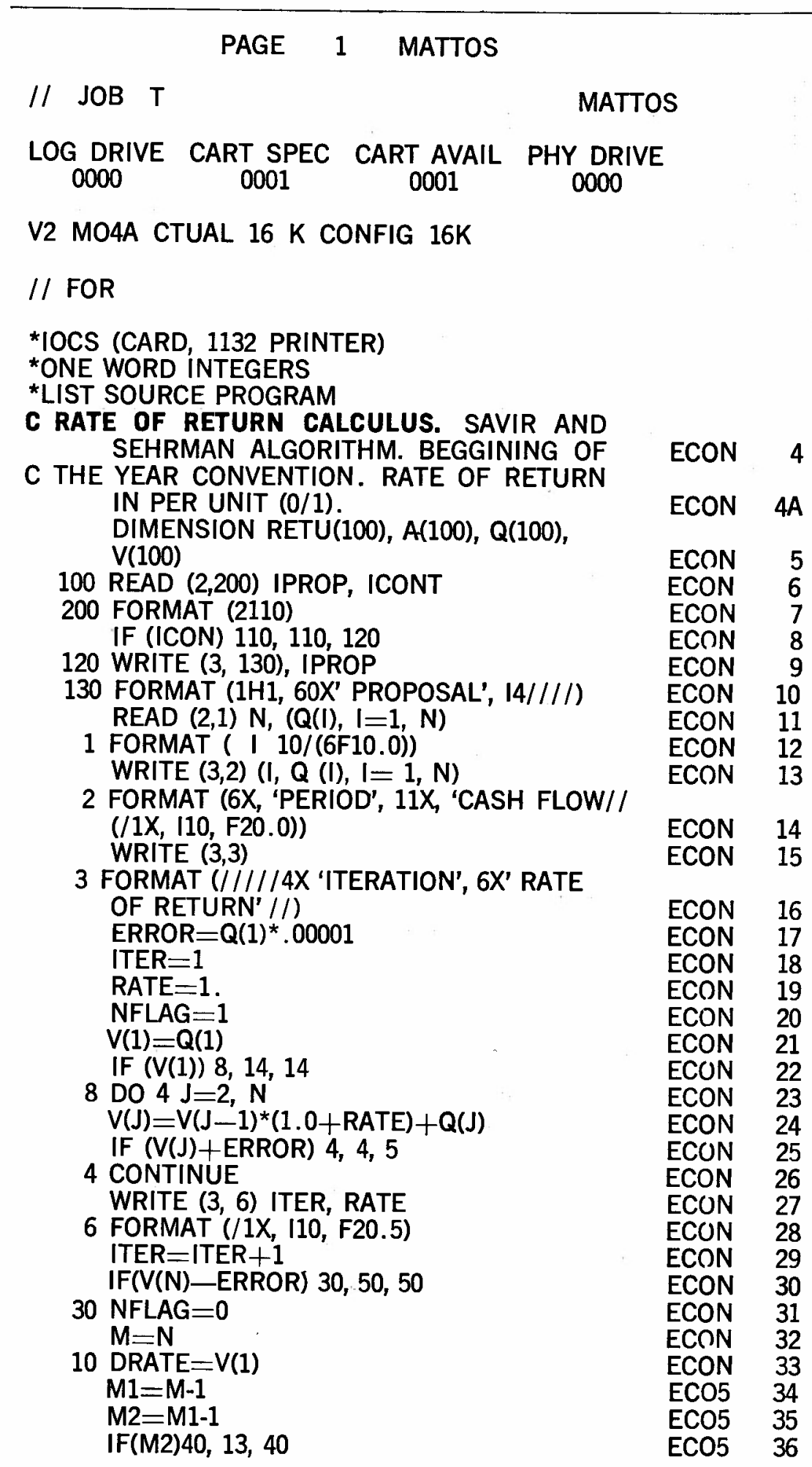




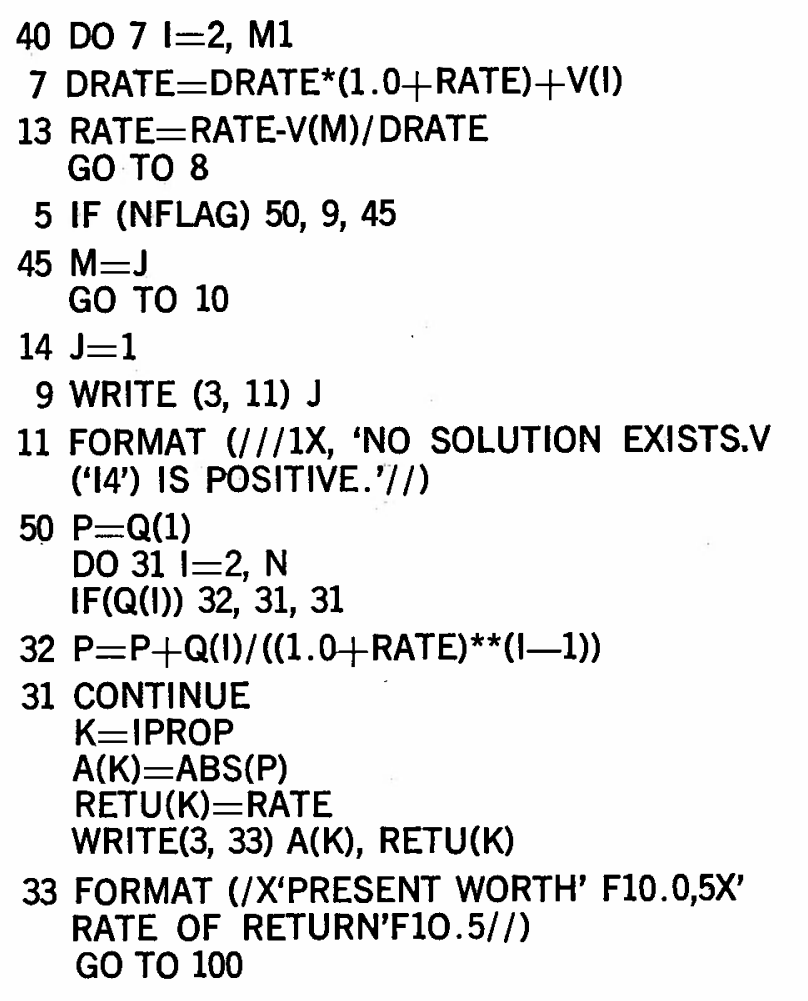

$\begin{array}{ll}\text { ECON } & 37 \\ \text { ECON } & 38 \\ \text { ECON } & 39 \\ \text { ECON } & 40 \\ \text { ECON } & 41 \\ \text { ECON } & 42 \\ \text { ECON } & 43 \\ \text { ECON } & 44 \\ \text { ECON } & 45 \\ & \\ \text { ECON } & 46 \\ \text { ECON } & 47 \\ \text { ECON } & 48 \\ \text { ECON } & 49 \\ \text { ECON } & 50 \\ \text { ECON } & 51 \\ \text { ECON } & 52 \\ \text { ECON } & 53 \\ \text { ECON } & 54 \\ \text { ECON } & 55 \\ \text { ECON } & 56 \\ \text { ECON } & 57\end{array}$

C CALCULUS OF THE MOST ECONOMICAL PROPOSAL. MATTOS ALGORITHM

$\begin{array}{ll}\text { ECON } & 58 \\ \text { ECON } & 59 \\ & \\ \text { ECON } & 60 \\ \text { ECON } & 61 \\ \text { ECON } & 62 \\ \text { ECON } & 63 \\ \text { ECON } & 64 \\ \text { ECON } & 65 \\ \text { ECON } & 66 \\ \text { ECON } & 67 \\ \text { ECON } & 68 \\ \text { ECON } & 69 \\ \text { ECON } & 70 \\ \text { ECON } & 71 \\ \text { ECON } & 72 \\ \text { ECON } & 73 \\ \text { ECON } & 74 \\ \text { ECON } & 75 \\ \text { ECON } & 76 \\ \text { ECON } & 77 \\ \text { ECON } & 78 \\ \text { ECON } & 79 \\ \text { ECON } & 80 \\ \text { ECON } & 81 \\ \text { ECON } & 82 \\ \text { ECON } & 83 \\ \text { ECON } & 84\end{array}$

110 WRITE $(3,39)$

39 FORMAT (1H1, 54X'BETTER PROPOSALS' //// 30X'PROPOSAL'19X'ALGORITHM' 119X'MINIMUM RETURN'//

$140 \operatorname{READ}(2,34)$ RMIN, JCONT

34 FORMAT (F10.5, 110) IF (JCONT)44, 44, 150

150 WRITE $(3,151)$ RMIN

151 FORMAT (/84XF10.5) DO $35 \mathrm{I}=1, \mathrm{~K}$

$35 \mathrm{~V}(\mathrm{I})=\mathrm{A}(\mathrm{I}) *(\mathrm{RETU}(\mathrm{l})-\mathrm{RMIN})$ DO 37 I=1,K

$37 \mathrm{Q}(\mathrm{I})=\mathrm{V}(\mathrm{I})$ DO $36 \mathrm{I}=1, \mathrm{~K}$ DO $36 \mathrm{~J}=\mathrm{I}, \mathrm{K}$ IF $(Q(I)-Q(J)) 38,36,36$

$38 \mathrm{~B}=\mathrm{Q}(\mathrm{J})$

$Q(J)=Q(I)$

$Q(I)=B$

36 CONTINUE DO $41 \mathrm{I}=1, \mathrm{~K}$ DO $41 \mathrm{~J}=1, \mathrm{~K}$ IF $(Q(I)-V(J)) 41,42,41$

42 WRITE $(3,43) \mathrm{J}, \mathrm{V}(\mathrm{J})$

43 FORMAT (30X, 14, 22X, F9.0)

41 CONTINUE GO TO 140

44 CALL EXIT END 


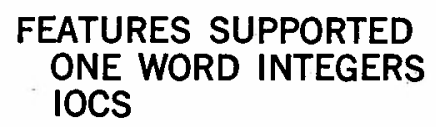

CORE REQUIREMENTS FOR

COMMON $O$ VARIABLES 830 PROGAM 770

END OF COMPILATION

// XEQ

\begin{tabular}{cr}
\hline PERIOD & CASH FLOW \\
1 & -100000 \\
2 & 8200 \\
3 & 8200 \\
4 & 8200 \\
5 & 8200 \\
6 & 8200 \\
7 & 8200 \\
8 & 8200 \\
9 & 8200 \\
10 & 8200 \\
11 & 108200
\end{tabular}

ITERATION RATE OF RETURN

$\begin{array}{rl}1 & 1.00000 \\ 2 & 0.80369 \\ 3 & 0.62828 \\ 4 & 4.47270 \\ 5 & 0.33718 \\ 6 & 0.22436 \\ 7 & 0.14087 \\ 8 & 0.09512 \\ 9 & 0.08277 \\ 10 & 0.08200 \\ 11 & 0.08200\end{array}$

PRESENT WORTH 100000.

RATE OF RETURN 0.08200

PERIOD CASH FLOW

$\begin{array}{rr}1 & -184000 \\ 2 & 31200 \\ 3 & 31200 \\ 4 & 31200 \\ 5 & 31200 \\ 6 & 31200 \\ 7 & 31200 \\ 8 & 31200 \\ 9 & 31200 \\ 10 & 31200 \\ 11 & 215200\end{array}$

ITERATION RATE OF RETURN

$\begin{array}{ll}1 & 1.00000 \\ 2 & 0.80802 \\ 3 & 0.63779 \\ 4 & 0.48894 \\ 5 & 0.36283 \\ 6 & 0.26385 \\ 7 & 0.19962 \\ 8 & 0.17345\end{array}$




$\begin{array}{ll}1 & 1.00000 \\ 2 & 0.72838 \\ 3 & 0.50606 \\ 4 & 0.33715 \\ 5 & 0.23205 \\ 6 & 0.19253 \\ 7 & 0.18764 \\ 8 & 0.18758\end{array}$

PRESENT WORTH 109000. RATE OF RETURN 0.18758

BETTER PROPOSALS

PROPOSAL ALGORITHM MINIALGORITHM MINI-
MUM
RETURN $\begin{array}{rr}5 & 12816 . \\ 1 & 1200 . \\ 4 & -1879 . \\ 3 & 75928 . \\ 2 & 16480 . \\ 5 & 11726 . \\ 1 & 200 . \\ 4 & -1931 . \\ 3 & 74928 . \\ 2 & 14640 . \\ 5 & 10636 . \\ 1 & -799 . \\ 4 & -1984 .\end{array}$

$\begin{array}{cc} & \\ & \\ 3 & 83928 . \\ 2 & 31200 . \\ 5 & 20446 . \\ 1 & 8200 . \\ 4 & -1510 . \\ 3 & 78928 . \\ 2 & 22000 . \\ 5 & 14996 . \\ 1 & 3200 . \\ 4 & -1774 . \\ & \\ 3 & 77928 . \\ 2 & 20160 . \\ 5 & 13906 . \\ 1 & 2200 . \\ 4 & -1826 . \\ 3 & 76928 . \\ 2 & 18320 .\end{array}$

0.00000

73928. 12800 . 9546. -1799 . $-2037$. 68928. 4096. 3600 . -2300 .

0.05000

$-6799$.

0.10000

0.15000

$\begin{array}{lr}3 & 63928 . \\ 5 & -1353 . \\ 4 & -2563 . \\ 2 & -5599 . \\ 1 & -11799 .\end{array}$

$\begin{array}{ll}3 & 63928 . \\ 5 & -1353 . \\ 4 & -2563 . \\ 2 & -5599 . \\ 1 & -11799 .\end{array}$

$\begin{array}{ll}3 & 63928 . \\ 5 & -1353 . \\ 4 & -2563 . \\ 2 & -5599 . \\ 1 & -11799 .\end{array}$

$\begin{array}{ll}3 & 63928 . \\ 5 & -1353 . \\ 4 & -2563 . \\ 2 & -5599 . \\ 1 & -11799 .\end{array}$

0.06000

$\begin{array}{ll}3 & 63928 . \\ 5 & -1353 . \\ 4 & -2563 . \\ 2 & -5599 . \\ 1 & -11799 .\end{array}$

0.20000 $\begin{array}{ll}4 & -6774 . \\ 3 & -16071 \\ 5 & -88553 \\ 1 & -91800 \\ 2 & -152800\end{array}$

\section{CONSIDERAÇOES FINAIS}

O critério de escolha, que apresentamos neste trabalho, deve ser considerado apenas como um auxílio na tomada de decisão do dirigente de emprêsa, e não como um critério absoluto.

De fato, como diz Grant, ${ }^{4}$ há vários fatôres econômicos que nem sempre podem ser quantificados: são os imponderáveis econômicos. Nos casos em que a diferença entre um plano e outro é pequena (dado por $V(j)$ nas considerações atrás), tais imponderáveis podem influir na decisão.
Salientamos, ainda, que tal critério, que desenvolvemos a partir das idéias de Grant, sòmente surtirá efeito desejado se fôr considerado o ciclo completo do investimento, isto é, tôdas as entradas e saídas de dinheiro, provenientes da utilização do plano, devem ser incluídas no fluxo de caixa, até a finalização completa do plano. Também não devem ser incluídas as saídas e entradas hipotéticas, como, por exemplo, a depreciação (que estará implícita no valor residual, êste incluído no último têrmo $Q(n)$ do fluxo de caixa da proposta em análise). 